OPEN ACCESS

Influence of the Binder on Lithium Ion Battery Electrode Tortuosity and Performance

To cite this article: Johannes Landesfeind et al 2018 J. Electrochem. Soc. 165 A1122

View the article online for updates and enhancements. 


\title{
Influence of the Binder on Lithium Ion Battery Electrode Tortuosity and Performance
}

\author{
Johannes Landesfeind, ${ }^{*, z}$ Askin Eldiven, and Hubert A. Gasteiger** \\ Chair of Technical Electrochemistry, Department of Chemistry and Catalysis Research Center, Technical University of \\ Munich, Munich, Germany
}

The electrochemical performance of porous graphite anodes in lithium ion battery applications is limited by the lithium ion concentration gradients in the liquid electrolyte, especially at high current densities and for thick coatings during battery charging. Beside the electrolyte transport parameters, the porosity and the tortuosity of the coating are key parameters that determine the electrode's suitability for high power applications. Here, we investigate the tortuosity of graphite anodes using two water as well as three $\mathrm{n}$-methyl-2-pyrrolidone based binder systems by analysis of symmetric cell impedance measurements, demonstrating that tortuosities ranging from $\sim 3-10$ are obtained for graphite anodes of similar thickness $(\sim 100 \mu \mathrm{m})$, porosities $(\sim 50 \%)$ and areal capacity $\left(\sim 3.4 \mathrm{mAh} / \mathrm{cm}^{2}\right)$. Furthermore, selected electrodes with tortuosities of 3.1, 4.3, and 10.2 were cycled in cells with reference electrode at charging C-rates from $0.1-20 \mathrm{1} / \mathrm{h}$, illustrating the clear correlation between electrode tortuosity and its rate capability. (C) The Author(s) 2018. Published by ECS. This is an open access article distributed under the terms of the Creative Commons Attribution Non-Commercial No Derivatives 4.0 License (CC BY-NC-ND, http://creativecommons.org/licenses/by-nc-nd/4.0/), which permits non-commercial reuse, distribution, and reproduction in any medium, provided the original work is not changed in any way and is properly cited. For permission for commercial reuse, please email: oa@electrochem.org. [DOI: 10.1149/2.0971805jes]

(c)) BY-NC-ND

Manuscript submitted February 2, 2018; revised manuscript received March 21, 2018. Published April 12, 2018. This was Paper 268 presented at the National Harbor, Maryland Meeting of the Society, October 1-5, 2017.

Understanding and predicting rate limitations in lithium ion batteries with porous electrodes requires profound knowledge of not only the electrolyte transport parameters (i.e., transference number, diffusion coefficient, conductivity) and the thermodynamic factor, but also the porosity and the tortuosity of the electrodes. The tortuosity of the electrode is particularly critical because the effective electrolyte conductivity and the effective diffusion coefficient in the electrolyte directly scale with the inverse of the tortuosity, so that care has to be taken to develop electrodes with minimized tortuosity. Using experimental approaches ${ }^{1-4}$ or $3 \mathrm{D}$ tomography, ${ }^{5,6}$ it was shown that the shape of active material particles distinctly influences the electrode tortuosity. For a given active material, however, electrode tortuosity and rate capability can also be improved by the design of the electrode layer such that short diffusion distances can be obtained across the electrode. For example, improved performance was demonstrated for graphite anodes when the platelet graphite particles were aligned normal to the current collector surface by means of a magnetic field ${ }^{7}$ or when silicon/graphite anode electrodes were laser structured. ${ }^{8}$ In this study we focus on the role of the electrode composition, specifically the role of the binder, on its tortuosity as well as on its implication for battery performance. While in the literature the link between binder and electrochemistry is frequently studied empirically using rate capability tests ${ }^{9}$ and long-term cycling experiments, ${ }^{10-13}$ we focus on the correlation of electrode tortuosity with binder content/type and its effect on rate capability.

In the following, the tortuosity of graphite anodes with different binders, different binder contents, and different amounts of conductive carbon additive will be determined by electrochemical impedance spectroscopy (EIS) of symmetric cells using the transmission line model approach. ${ }^{3,14}$ In the first part of our analysis we will demonstrate the effect of binder and conductive carbon additives on electrode tortuosity and thereafter give an overview of the range of experimentally obtained tortuosities for two water and three NMP (n-methyl-2-pyrrolidone) based binder systems. Subsequently, charging rate performance tests in three-electrode cells for electrodes with largely different tortuosities are presented, illustrating the clear correlation between the tortuosity of the anode electrode and its rate capability.

\footnotetext{
*Electrochemical Society Student Member

**Electrochemical Society Fellow.

${ }^{\mathrm{z} E}$-mail: j.landesfeind@tum.de
}

\section{Experimental}

Electrode preparation.-Composite slurries of graphite, binder, conductive carbon additive (material parameters and abbreviations given in Table I) and NMP (Sigma Aldrich, anhydrous, 99.5\%) were prepared in a planetary mixer (Thinky ARV-310). As the mixing process is not at the focus of the current study, all solid components and half of the solvent were filled into the mixer at once and mixed at $2000 \mathrm{rpm}$ for at least $2 \mathrm{~min}$. The remaining solvent was added stepwise between subsequent mixing steps until similar slurry viscosities are obtained, as judged by eye. The prepared slurries were coated onto a copper current collector foil (MTI, $9 \mu \mathrm{m}$ ) using the doctor blade technique with a wet-film thickness of $200 \mu \mathrm{m}$. Coatings were dried in a self-built drying oven at $50^{\circ} \mathrm{C}$ under air and then punched (Hohsen Corporation) to circular discs of $11 \mathrm{~mm}$ diameter $\left(A_{\mathrm{El}}=0.95 \mathrm{~cm}^{2}\right)$ before assembly in T-Cells without prior compression. Areal loading of the all graphite electrodes (thickness $98 \pm 13 \mu \mathrm{m}$ ) used in this work were $9.6 \pm 1.8 \mathrm{mg} \mathrm{Gra}_{\mathrm{G}} / \mathrm{cm}^{2}$ (corresponding to $3.4 \pm 0.6 \mathrm{mAh} / \mathrm{cm}^{2}$ or $3.2 \pm 0.6 \mathrm{mAh} / \mathrm{El}$. if referenced to the total electrode, using a theoretical graphite intercalation capacity of $350 \mathrm{mAh} / \mathrm{cm}^{2}$ ).

Tortuosity determination.-Tortuosities were determined for two nominally identical cells at each composition, using the same experimental procedure as described before (compare Experimental section in Ref. 3) unless stated otherwise. A 1:1 (by weight) mixture of ethylene carbonate (EC, Sigma Aldrich, anhydrous, 99\%) and diethyl carbonate (DEC, Sigma Aldrich, anhydrous, >99\%), was used as solvent for self-prepared electrolytes containing $12 \mathrm{mM}$ tetrabutylammonium perchlorate $\left(\mathrm{TBAClO}_{4}\right.$, Sigma Aldrich, $\left.\geq 99.0 \%\right)$ salt. A turn-key conductivity sensor (LF $1100+$, SI Analytics, with custom-made ground glass fitting) with a built-in temperature sensor was used to determine the electrolyte conductivity (equating to $423 \mu \mathrm{S} / \mathrm{cm}$ at $25^{\circ} \mathrm{C}$ ). Symmetrical Swagelok type T-Cells (spring-compressed to $\approx 1$ bar) were built outside the glove box, then transferred into a temperature controlled climate chamber $\left(25^{\circ} \mathrm{C}\right.$, Binder $)$ and impedance spectra were recorded (Biologic VMP3) around the open circuit potential (OCV) after a resting period of at least $12 \mathrm{~h}$ in a frequency range of $200 \mathrm{kHz}$ to $0.1 \mathrm{~Hz}$ with a $20 \mathrm{mV}$ perturbation. No artifacts from the cell assembly under air were observed, which was confirmed by monitoring the electrolyte conductivity (no measurable change over period of 6 months) as well as by comparative measurements with cells assembled inside the glove box. 
Table I. List of the materials used for the preparation of porous graphite anodes, including supplier specifications, their BET surface area, and their density (* from manufacturer's specification).

\begin{tabular}{|c|c|c|c|}
\hline Material & Abbreviation & $\begin{array}{l}\text { BET Surface Area } \\
\mathrm{m}^{2} / \mathrm{g}\end{array}$ & $\begin{array}{c}\begin{array}{c}\text { Density } \\
\mathrm{g} / \mathrm{cm}^{3}\end{array} \\
\end{array}$ \\
\hline Graphite, T311, Timcal, $19 \mu \mathrm{m}$ D50 & Gra & $3^{*}$ & 2.26 \\
\hline SuperC65, Timcal & C65 & $62^{*}$ & 2.26 \\
\hline Styrene-Butadiene Rubber, MTI & SBR & - & 0.94 \\
\hline Alginic acid sodium salt, Sigma Aldrich & Alginate & - & 1.60 \\
\hline PVDF, KF1100, homopolymer, Kureha & Kureha & - & $1.78^{*}$ \\
\hline
\end{tabular}

Cycling experiments.-For charging rate performance measurements of selected composite graphite electrodes, for each composition three three-electrode Swagelok T-cells utilizing a lithium reference and a lithium counter electrode (11 mm diameter; $450 \mu \mathrm{m}$ thickness, Rockwood Lithium) were built inside an argon filled glove box (MBraun, $25^{\circ} \mathrm{C} \pm 1{ }^{\circ} \mathrm{C}$, oxygen and water content $<0.1 \mathrm{ppm}$, Ar 5.0, Westfalen, $99.999 \%$ vol.). All cell parts were cleaned thoroughly by boiling them in an ethanol water mixture, rinsing them with water (Millipore, Elix, $15 \mathrm{M} \Omega$ ), and then drying them at $70^{\circ} \mathrm{C}$ in a drying oven before bringing them into the glove box. Two porous glass fiber separators (VWR, $250 \mu \mathrm{m}$ thickness) and a commercially available battery electrolyte (1 M LiPF 6 in EC:EMC 3:7, w:w, LP57, battery grade, BASF) were used for the cycling experiment in a temperature controlled climate chamber $\left(25^{\circ} \mathrm{C}\right.$, Binder $)$ outside the glove box using a potentiostat (BioLogic, VMP3). Without any additional formation cycle, three consecutive galvanostatic charging (i.e., lithiation) cycles with a lower cutoff potential of $5 \mathrm{mV}$ versus the lithium reference electrode were recorded. For the charging and the discharging currents of the graphite electrodes a capacity value of $3 \mathrm{mAh} / \mathrm{El}$. was used for all electrodes, corresponding to intercalation currents of 0.3 , $0.6,1.5,3,6,15,30$ and $60 \mathrm{~mA} / \mathrm{El}$., corresponding to approximate C-rates of $0.1,0.2,0.5,1,2,5,10,201 / \mathrm{h}$ (within $\pm 10 \%$ due to minor loading variations), while the discharge (i.e., delithiation) current was held constant at $0.3 \mathrm{~mA} / \mathrm{El} .(\sim 0.11 / \mathrm{h})$, with an upper cutoff potential of $1 \mathrm{~V}$ vs. the lithium reference electrode. For the sake of simplicity, the rounded C-rates are used in the text for reference, and the charging capacities of the third cycle at $0.11 / \mathrm{h}$, given in Table II are used to calculate the exact C-rates used for the analysis shown in Figure 4 and Figure 5.

\section{Results and Discussion}

In the following, the effect of electrode composition on the tortuosity of porous graphite electrodes with different mass ratios of conductive additive (SuperC65) and binder will be demonstrated exemplarily with PVDF based graphite anodes. Figure 1 shows the tortuosities of different anode compositions, obtained from the transmission line analysis of the EIS response of symmetric cell measurements (details about this analysis technique and the tortuosity calculations can be found elsewhere $\left.\mathrm{r}^{3,14}\right)$. Dashed lines in Figure 1 serve as guide-to-theeye only; error bars indicate the error introduced by the electrode thickness measurement $(2 \mu \mathrm{m})$ and the electrolyte conductivity (1\%) and for all measurements two nominally identical cells yield very reproducible results.

At first we will discuss the results for anodes with only active material and Kureha PVDF binder, prepared as described in the Experimental section. For binder weight percentages of 1.5, 3, 6 and $10 \mathrm{wt} \%$, the tortuosity values increase linearly from $2.8 \pm 0.1$ to $5.2 \pm 0.2$ (compare Figure 1a, green crosses), an enormous, nearly 2 -fold increase, although the electrode composition was altered only by $8.5 \mathrm{wt} \%$ ( $=10 \mathrm{wt} \%-1.5 \mathrm{wt} \%$ ). This is the more surprising, as all electrodes tested here are uncompressed and thus have very similar porosities $(51 \pm 5 \%)$ and are based on the same active material, so that one would expect little changes in tortuosity. The observed large tortuosity changes therefore suggest that the binder content strongly affects the effective transport properties in the electrolyte phases within the porous electrode, i.e., diffusion coefficient and conductivity. It is clear, of course, that the chosen binder weight ratios span a rather extreme range, as electrodes with only $1.5 \mathrm{wt} \%$ of the Kureha PVDF are very brittle and have to be handled carefully, while $10 \mathrm{wt} \%$ binder would substantially decrease the energy density of the electrodes and could clog the pores for electrolyte transport. ${ }^{15}$

Based on empirical studies by Marks et al., the best electrochemical performance while still maintaining good mechanical stability is obtained with an average binder layer thickness of $\approx 6 \mathrm{~nm}$. ${ }^{15}$ The binder layer thickness $d_{\mathrm{Bi}}$ corresponds to the thickness of the binder layer, if the binder were homogeneously distributed over the BET surface areas of active material $\left(\mathrm{BET}_{\mathrm{Gra}}\right)$ and the conductive carbon additives $\left(\mathrm{BET}_{\mathrm{C} 65}\right.$; see BET values given in Table $\left.\mathrm{I}\right)$, and may be calculated by

$$
d_{\mathrm{Bi}}=\frac{\sum\left(\frac{m_{\mathrm{Bi}}}{\rho_{\mathrm{Bi}}}\right)}{m_{\mathrm{Gra}} \cdot \mathrm{BET}_{\mathrm{Gra}}+m_{\mathrm{C} 65} \cdot \mathrm{BET}_{\mathrm{C} 65}}
$$

where $\mathrm{m}_{\mathrm{i}}$ represents the mass of component $\mathrm{i}$ per $100 \mathrm{~g}$ of electrode and $\rho_{\mathrm{Bi}}$ is the density of the binder $(\mathrm{Bi}=\mathrm{CMC} / \mathrm{SBR} /$ Kynar/Solef/Alginate/Kureha, values given in Table I). Due to the low BET surface area of typical graphite active materials (generally

\footnotetext{
Table II. Compositions and parameters of graphite anodes used for charging rate performance tests in three-electrode cells with metallic lithium counter and reference electrodes. Porosity, thickness and tortuosity values are taken from electrodes of the same coating which were used for the tortuosity measurement (errors indicate the standard deviation of 2 cells). Loading values are calculated based on the mass and composition of the electrodes used for the cycling experiments, using a theoretical intercalation capacity of $350 \mathrm{mAh} / \mathrm{g}_{\mathrm{Gra}}$. The charging capacity (i.e., the lithiation capacity) is given at the $3^{\text {rd }}$ cycle at a C-rate of $0.11 / \mathrm{h}$ and for a cutoff voltage of $5 \mathrm{mV}$ vs. the lithium reference electrode potential, the error bar describes the standard deviation of three cells.
}

\begin{tabular}{|c|c|c|c|c|c|c|}
\hline $\begin{array}{c}\text { Electrode Composition } \\
\text { Gra/Bi/C65 } \\
\text { wt } \%\end{array}$ & Binder & $\begin{array}{c}\text { Porosity } \\
\%\end{array}$ & $\begin{array}{c}\text { Thickness } \\
\mu \mathrm{m}\end{array}$ & $\begin{array}{c}\text { Loading } \\
\mathrm{mg}_{\mathrm{Gra}} / \mathrm{cm}^{2}\end{array}$ & $\begin{array}{c}Q_{\text {charging }} \text { at } 3^{\text {rd }} \\
0.1 \mathrm{C} \mathrm{cycle} \\
\mathrm{mAh} / \mathrm{cm}^{2}\end{array}$ & $\begin{array}{c}\text { Tortuosity } \\
-\end{array}$ \\
\hline $97 / 3 / 0$ & Alginate & $56 \pm 1$ & $100 \pm 2$ & 9.8 & $3.20 \pm 0.13$ & $3.1 \pm 0.1$ \\
\hline $97 / 3 / 0$ & Kynar & $52 \pm 2$ & $95 \pm 2$ & 9.4 & $3.44 \pm 0.21$ & $4.3 \pm 0.1$ \\
\hline $94 / 6 / 0$ & CMC/SBR $1: 1$ & $46 \pm 1$ & $101 \pm 5$ & 10.2 & $3.57 \pm 0.21$ & $10.2 \pm 0.3$ \\
\hline
\end{tabular}





Figure 1. Tortuosities of graphite electrodes obtained from symmetric cell EIS measurements with (orange) or without (green) conductive carbon C65 plotted versus a) the binder mass percentage and b) the average binder layer thickness (according to Eq. 1). Exact electrode compositions of graphite active material (Gra), binder (Bi), and conductive carbon additive (C65) are given in terms of mass percentages in the figures using the nomenclature $\mathrm{Gra} / \mathrm{Bi} / \mathrm{C} 65$. Dashed lines serve as guide-to-the-eye only; error bars indicate the tortuosity uncertainty due to finite accuracy of the thickness $(2 \mu \mathrm{m})$ and conductivity (1\%) measurements, two measurements are shown for each composition.

in order to minimize irreversible SEI formation losses), really high binder layer thickness values are normally only obtained for composite electrodes of binder and active material only, i.e., without conductive carbon additives. This is illustrated in Figure 1b, which represents the same data as in Figure 1a, but re-scaled in terms of binder layer thickness rather than binder weight percentage. For electrodes without any conductive carbon additive (green crosses in Figure 1b), the binder layer thickness ranges from 2.9 to $20.8 \mathrm{~nm}$, much higher than the reported sweet spot of $6 \mathrm{~nm} .{ }^{15}$ Closest to the suggested optimal $\approx 6 \mathrm{~nm}$ binder layer thickness are the electrodes with $3 \mathrm{wt} \%$ binder.

Also shown in Figure 1 are the tortuosities of three electrode compositions (see figure for exact compositions) which additionally include different amounts of the conductive carbon C65. These electrodes were prepared by replacing a fraction of the active material of the $6 \mathrm{wt} \%$ and $10 \mathrm{wt} \%$ binder electrodes by $\mathrm{C} 65$ so that one obtains the same average binder layer thickness value of the electrodes without $\mathrm{C} 65$ and 3 wt $\%$ binder have $(5.8 \mathrm{~nm}$, see Figure 1b). Analogously electrodes with $3 \mathrm{wt} \%$ binder and C65 were prepared to obtain the same binder layer thickness of $2.9 \mathrm{~nm}$ as for the $1.5 \mathrm{wt} \%$ binder electrodes without C65. In all these cases, due to the high surface area of C65, only a small mass fraction of C65 is necessary to adjust the binder layer thickness, and the resulting compositions are given in the figure. Figure 1a shows that the C65 containing electrodes with $6 \mathrm{wt} \%$ and $10 \mathrm{wt} \%$ binder yield much lower tortuosity values compared to their C65 free counterparts. With $10 \mathrm{wt} \%$ Kureha PVDF binder, the tortuosity decreases from $5.2 \pm 0.2$ to $3.3 \pm 0.1$ by tuning the average binder layer thickness to $5.8 \mathrm{~nm}$ by addition of high surface area C65. Similarly, with $6 \mathrm{wt} \%$ binder the tortuosity decreases from $3.9 \pm 0.1$ to $3.5 \pm 0.1$ by C65 addition (compare Figure 1a). In terms of binder layer thickness, Figure $1 \mathrm{~b}$ shows that all electrodes with a binder layer thickness of $5.8 \mathrm{~nm}$, with or without C65 have similar tortuosities. This suggests, that the calculated binder layer thickness serves as a better indicator for the tortuosity of the electrodes than the binder amount, and that the high surface area conductive carbon additive behaves like a sponge, taking up a large fraction of the binder. By addition of C65 the binder layer thickness is reduced and (partially) blocked ionic conduction paths might become accessible, thereby reducing the coatings tortuosity. For the examples discussed so far, the electrode tortuosity increases with the average binder thickness (as calculated by Eq. 1), irrespective of whether conductive carbon is part of the electrode formulation (see Figure $1 \mathrm{~b}$ ). The nearly 2fold tortuosity increase between the electrodes with $1.5 \mathrm{wt} \%$ binder $(\mathrm{Gra} / \mathrm{Bi} / \mathrm{C} 65=98.5 / 1.5 / 0$, s. Figure $1 \mathrm{~b})$ and $10 \mathrm{wt} \%$ binder ( $\mathrm{Gra} / \mathrm{Bi} / \mathrm{C} 65=95 / 10 / 0$, s. Figure $1 \mathrm{~b})$ is quite striking, since even for the highest binder content of $10 \mathrm{wt} \%$ the volume fraction of the binder $\left(\varepsilon_{\text {binder }} \approx 6 \%\right)$ is still nearly an order of magnitude lower than the void volume fraction of the electrode $\left(\varepsilon_{\text {void }} \approx 51 \pm 5 \%\right)$. Quite evidently, even small volume fractions of binder strongly affect electrode tortuosity.

The clear trend between average binder thickness and tortuosity, however, is not found for the $3 \mathrm{wt} \%$ binder electrodes, for which the tortuosity increases from $3.2 \pm 0.1$ to $3.6 \pm 0.1$ when $\mathrm{C} 65$ is added to obtain a binder layer thickness reduction from 5.8 to $2.9 \mathrm{~nm}$. Because both measurements for the composition 91.9:3:5.1 (Gra/Bi/C65, by weight) were done using electrodes from the same coating, these measurements were repeated using a newly prepared coating. However, also these repeat measurements yielded the same higher tortuosities compared to the C65 free electrodes using the same amount of $3 \mathrm{wt} \%$ binder. This trend is in agreement with our previous publication for porous LFP cathodes at $\sim 45 \%$ porosity, (Ref. 3), where we reported higher tortuosities of $\sim 5$ with $5 \mathrm{wt} \%$ binder and 5 wt $\%$ C65 corresponding to a small average binder layer thickness of $1.2 \mathrm{~nm}$, in contrast to a tortuosity of only $\sim 3.5$ with $15 \mathrm{wt} \%$ binder and $15 \mathrm{wt} \%$ C65 corresponding to a binder layer thickness of $3.3 \mathrm{~nm}$. Thus, similar to the observations made with the graphite electrodes with low average binder layer thickness, C65 addition and the accompanying average binder layer thickness reduction leads to an increase in tortuosity. Currently we have no explanation of the detrimental effect from C65 addition on the electrode tortuosity for resulting average binder layer thicknesses of $<<6 \mathrm{~nm}$, and further investigations are necessary to check for example for an inhomogeneous carbon and binder distribution, ${ }^{16}$ which was shown to negatively affect the C-rate capability of lithium ion battery anodes, ${ }^{9}$ which we believe results from a lowering of the electrode tortuosity.

In addition to the presented binder layer thickness versus tortuosity study utilizing the Kureha PVDF binder, four other binders were analyzed, namely two PVDF binders from different suppliers for use with NMP based inks, as well as CMC/SBR (here always in 1:1 mass ratio) and alginate for use with water based inks. This comparison was conducted with electrodes without conductive carbon and various amounts of binder content $(1.5,3,6$, and $10 \mathrm{wt} \%$ for the Kureha PVDF and the CMC/SBR (1:1) binder, as well as 3 and $6 \mathrm{wt} \%$ for the Kynar PVDF, Solef PVDF, and alginate binder). The electrode tortuosities obtained by the transmission line model EIS analysis are shown in Figure 2 versus the average binder thickness calculated from Eq. 1, whereby crosses and dashed lines indicate NMP based PVDF 


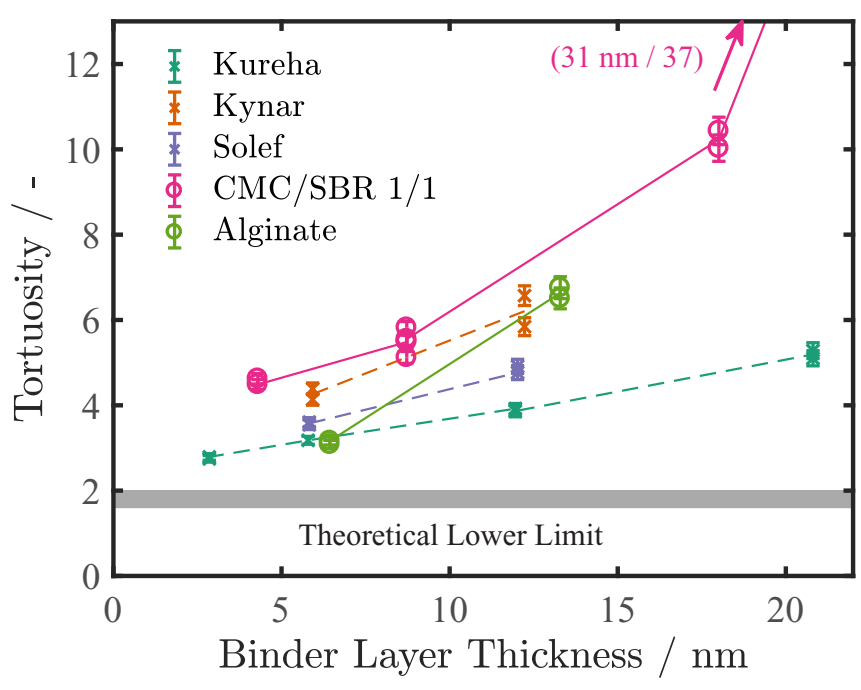

Figure 2. Tortuosities of porous graphite electrodes without conductive carbon and with different amounts of NMP based (Kureha, Kynar, Solef PVDFs; dashed lines) and water based (CMC/SBR and alginate; solid lines) binders, plotted versus the average binder thickness determined by Eq. 1. The binder contents were $1.5,3,6$, and $10 \mathrm{wt} \%$ for the Kureha PVDF and the CMC/SBR (1:1) binder, as well as 3 and $6 \mathrm{wt} \%$ for the Kynar PVDF, Solef PVDF, and alginate binder. Tortuosities were measured using the transmission line model EIS analysis in symmetric cell configuration with $12 \mathrm{mM} \mathrm{TBAClO}{ }_{4}$ in EC:DEC (1:1 w:w) (see Ref. 3 for details). Lines serve as a guide-to-the-eye only; error bars indicate the tortuosity uncertainty stemming from the finite accuracy of the thickness $(2 \mu \mathrm{m})$ and conductivity $(1 \%)$ measurements, two measurements are shown for each composition.; the gray region gives an estimate for the likely lower tortuosity limit based analysis of X-ray tomography data (see text).

electrodes, while circles and solid lines represent the water based systems. The first thing to notice is that all porous graphite electrodes show the same general trend, viz., lower tortuosities for smaller amounts of binder. The measured tortuosities range from $2.7 \pm 0.1$ (Kureha, $1.5 \mathrm{wt} \%$ binder) to $10.2 \pm 0.3$ (CMC/SBR 1:1, $6 \mathrm{wt} \%$ binder); for $10 \mathrm{w} . \%$ of the CMC/SBR binder, the tortuosity increases to an extremely high value of 37 (indicated by the pink arrow in Figure 2). The gray rectangle in Figure 2 depicts the tortuosity range for platelet graphite obtained using X-ray tomography for the range of porosities used in this work $(51 \pm 5 \%)$, which may be considered to be the theoretical lower tortuosity limit for the graphite active material if unaffected by the binder, as X-ray tomography cannot resolve the binder/conductive carbon phase and thus commonly ignores its effect on tortuosity. ${ }^{5,14}$ By an approximate extrapolation of the tortuosities from the presented EIS measurements in Figure 2 to $0 \mathrm{wt} \%$ binder, limiting tortuosity values for conceptually binder-free anodes between 2.4 and 4 can be projected, which is reasonably close to the tortuosity range for electrodes estimated from X-ray tomography (1.6 to 2.0), where the binder contribution is not considered. The remaining difference might be due to different aspect ratios of the graphite particles or to the inability to sufficiently extrapolate from 1.5-3 wt $\%$ binder to $0 \mathrm{wt} \%$ binder.

As proposed in the literature,,${ }^{15}$ electrodes with a binder layer thickness of 5-10 nm have the highest relevance for practical consideration, representing a trade-off between sufficient adhesion and maximized active material content (see above), for which reason we will focus our comparison to graphite anodes with $3 \mathrm{wt} \%$ binder, corresponding to average binder film thicknesses of $\sim 6-9 \mathrm{~nm}$ (this variation is due to the variation in binder density, see Table I). For this binder content, the lowest tortuosity is found for alginate $(3.1 \pm 0.1)$ and the highest tortuosity for CMC/SBR 1:1 (5.5 \pm 0.1$)$ binder. Even for electrodes with the same binder type (PVDF) with albeit different functionalized groups and molecular weights, the tortuosities differ substantially: $3.2 \pm 0.1$ for the Kureha PVDF binder, $3.6 \pm 0.1$ for the PVDF binder

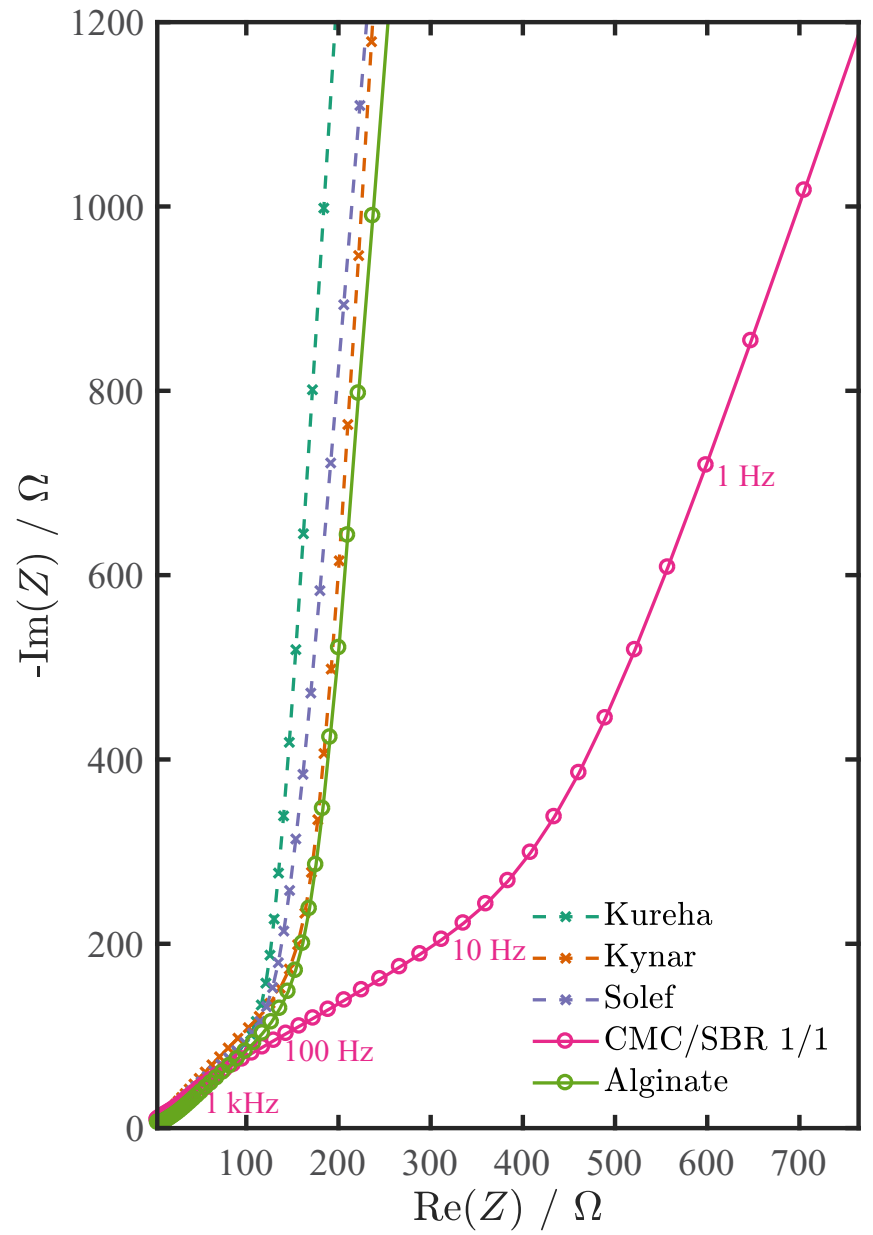

Figure 3. Nyquist plots of symmetric cells using graphite electrodes without conductive carbon additive and with $6 \mathrm{wt} \%$ water based (solid lines, circles) and NMP based (dashed lines, crosses) binders. The EIS spectra are shifted to the origin for better comparability. A non-intercalating electrolyte with an ionic conductivity of $0.423 \mathrm{mS} / \mathrm{cm}$ was used $\left(12 \mathrm{mM} \mathrm{TBAClO}_{4}\right.$ in EC:DEC $1: 1)$ and impedance measurements were recorded from $200 \mathrm{kHz}$ to $0.1 \mathrm{~Hz}$ with an excitation amplitude of $20 \mathrm{mV}$. The pink numbers next to the CMC/SBR data mark the frequencies in $\mathrm{Hz}$ at which the data were taken; lines serve as a guide-to-the-eye only.

from Solvey, and $4.3 \pm 0.1$ for the PVDF binder from Kureha. Quite strikingly, the here prepared graphite electrodes with the CMC/SBR binder system show much larger ionic resistances compared to the other binder systems, especially for $6 \mathrm{wt} \%$ and $10 \mathrm{wt} \%$ binder (compare pink circles in Figure 2). While the average binder film thickness for the electrode with $6 \mathrm{wt} \% \mathrm{CMC} / \mathrm{SBR}(\sim 18 \mathrm{~nm})$ is even lower than that for the electrode with $10 \mathrm{wt} \%$ Kureha PVDF binder $(\sim 21 \mathrm{~nm})$, the tortuosity of the former $(10.2 \pm 0.3)$ is substantially higher than that of the latter $(5.2 \pm 0.1)$. This could be due to an inhomogeneous distribution of the CMC/SBR binder across the electrode, leading to either the formation of a binder-rich surface layer/film at the electrode surface blocking ionic transport or to blocking the electronic pathway across the electrode, which would invalidate the impedance analysis (in the here used transmission line model, the electronic resistance across the electrode is assumed to be low compared to the ionic resistance, which typically would be expected for graphite anodes). ${ }^{14}$

Further insights might be gained by a closer inspection of the Nyquist plots for graphite anodes with $6 \mathrm{wt} \%$ binder, which is shown in Figure 3 (note that the EIS response is shifted to the origin for better comparability). The unique behavior of the CMC/SBR electrodes is reflected by the apparent inflections of its EIS spectrum (pink circles) in the mid frequency region $(100-10 \mathrm{~Hz})$ as well as 
in the low frequency region $(10-0.1 \mathrm{~Hz})$, in contrast to the nearly straight 45-degree lines for the electrodes with the other binders. This feature for the CMC/SBR electrodes, together with the much higher apparent tortuosity values may be caused by an inhomogeneous distribution of the binder, ${ }^{17}$ which may result from the non-optimized slurry mixing and electrode drying steps. ${ }^{18}$ Even for highly conductive graphite particles the required assumption for the applicability of the simplified transmission line model (i.e., neglecting the solid phase electronic resistance) might be erroneous if the binder agglomerates and/or is distributed inhomogeneously. As long as the electronic resistance is minor, the EIS tortuosity measurement is invariant toward ionic conductivity, ${ }^{14}$ however repeat measurements for the electrodes with $6 \mathrm{wt} \% \mathrm{CMC} / \mathrm{SBR}$ with a higher electrolyte conductivity (2.5 instead of $0.423 \mathrm{mS} / \mathrm{cm}$ ) yield much higher values for the tortuosity ( $>100$ vs. $\sim 10)$. Although, with optimized electrode preparation, the absolute values for the CMC/SBR binder system in this work may not be representative. Nevertheless, these electrodes demonstrate the sensitivity of electrode preparation and its pronounced effect on the effective ionic and electronic transport properties.

In summary Figure 2 clearly shows the strong dependence of the tortuosity of porous anodes not only on the binder content and the average binder layer thickness, but also on the type of binder. Again, the reader is reminded that the here prepared electrodes have not been optimized in any way (i.e., the same mixing and drying procedures were used for all binders). Therefore, while we believe that the generally observed trend of increasing tortuosity with increasing binder content is correct for each binder, our data do not allow to make a quantitative comparison and judgement between the tortuosities obtained with different binders and dispersing agents. It is emphasized that remarkably different tortuosity values $(\sim 3-10)$ are obtained for the graphite anodes under study although the binder volume fraction is minor (here between 0.8 and $5 \%$ for binder mass fractions of $1.5 \mathrm{wt} \%$ to $10 \mathrm{wt} \%$ ) compared to the void volume fraction of the electrodes (here $51 \pm 5 \%$ ). The pronounced effect of the small volume fraction binder phase was previously shown for inhomogeneous binder distributions prepared by different drying procedures. ${ }^{9}$ More detailed investigations of the electrode preparation steps and their influence on electrode tortuosity will be subject of a future work. ${ }^{18}$

Electrochemical performance with different EIS tortuosities.After having determined the tortuosity value of the various electrodes, i.e., the scaling factor of the effective diffusion coefficient and the effective ionic conductivity, ${ }^{19}$ we will now examine experimentally the relationship between tortuosity and electrochemical performance. To verify the expected behavior of better performance for low tortuosity electrodes, ${ }^{20,21}$ we picked three electrode compositions with different tortuosities but similar areal capacity, thickness, and porosity in order to compare their electrochemical performance and their rate capability using three-electrode cells with a lithium counter and a lithium reference electrode. As the limiting operation mode for graphite anodes is the charging step, due to the proximity of the graphite intercalation potential to the lithium plating potential, we focus our analysis on the charging (i.e., lithiation) behavior of graphite anodes. From the set of the above investigated electrodes, electrodes with tortuosities of $3.1 \pm 0.1,4.3 \pm 0.1$, and $10.2 \pm 0.3$ were selected for electrochemical performance tests (the corresponding electrode compositions and parameters are given in Table II). Again, it is important to note that all electrodes used in the following rate tests were uncompressed and had very comparable thicknesses of $98 \pm 3 \mu \mathrm{m}$ and porosities of $51 \pm 5 \%$. For details on the rate test measurement procedure the reader is referred to the Experimental section.

Figures $4 \mathrm{a}$ and $4 \mathrm{~b}$ show the galvanostatic charging curves for the $3^{\text {rd }}$ cycle at a C-rate of $0.11 / \mathrm{h}$ and $11 / \mathrm{h}$ of graphite anodes with different tortuosities. The charging curves were measured versus the lithium reference electrode and are normalized to their charging capacity obtained in the $3^{\text {rd }}$ cycle at $0.11 / \mathrm{h}$ (compare Figure $4 \mathrm{a}$ ), which were essentially the same $\left(3.4 \pm 0.2 \mathrm{mAh} / \mathrm{cm}^{2}\right.$, see Table II). In stark contrast to the behavior at low charging rate, at a C-rate of $11 / \mathrm{h}$ (compare Figure $4 \mathrm{~b}$ ) the highest capacity (given in terms of
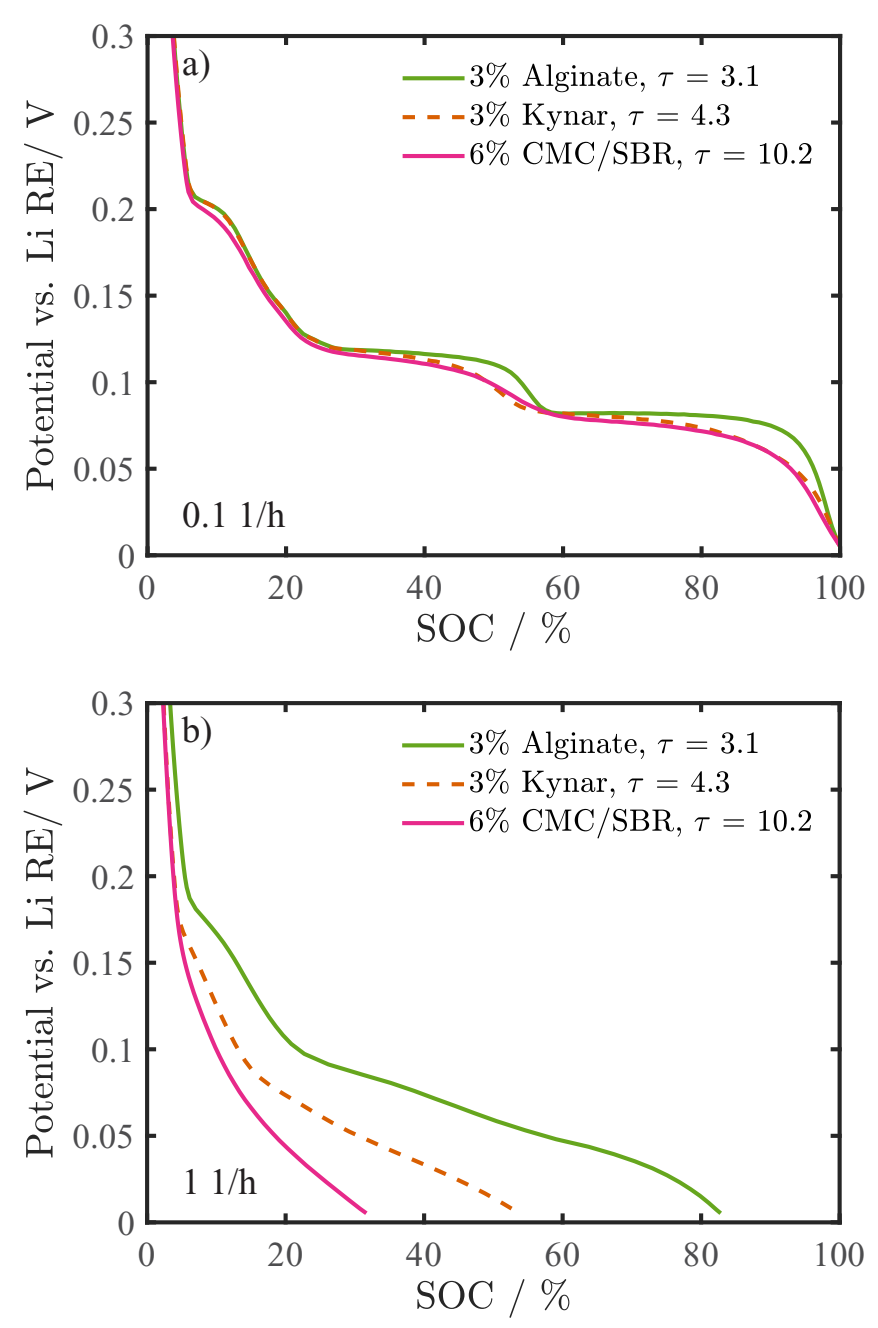

Figure 4. Third-cycle galvanostatic charging (lithiation) curves at C-rates of a) $0.11 / \mathrm{h}$ and b) $1 \mathrm{1} / \mathrm{h}$ of porous graphite electrodes (see Table II for details) with three different tortuosities, recorded in three-electrode cells with lithium counter and lithium reference electrodes. Measurement were conducted using $1 \mathrm{M} \mathrm{LiPF}_{6}$ in EC:EMC $(3: 7 \mathrm{w}: \mathrm{w})$ as an electrolyte and at $25^{\circ} \mathrm{C}$. The upper and lower cutoff potentials were $1 \mathrm{~V}$ and $5 \mathrm{mV}$ vs. the metallic lithium reference electrode, respectively. Capacities are normalized to the third-cycle capacity at $0.1 \mathrm{1} / \mathrm{h} \mathrm{(a)}$; they are all within $3.4 \pm 0.2 \mathrm{mAh} / \mathrm{cm}^{2}$, and are given in terms of state-of-charge (SOC). Table II gives all parameters of the used electrodes.

state-of-charge, SOC) is reached for the alginate based electrodes with $3 \mathrm{wt} \%$ binder and the lowest tortuosity $(\tau=3.1 \pm 0.1)$, for which more than $80 \%$ of the lithiation capacity can be obtained until a cutoff potential of $5 \mathrm{mV}$ (vs. the lithium reference electrode) is reached. For the $3 \mathrm{wt} \%$ Kynar PVDF electrodes $(\tau=4.3 \pm 0.1)$, only $50 \%$ of the full capacity is accessible until the $5 \mathrm{mV}$ cutoff potential is reached, while for the high tortuosity $(10.2 \pm 0.3) \mathrm{CMC} / \mathrm{SBR}$ based electrodes the capacity is limited to only $30 \%$ SOC. Thus, the accessible lithiation capacities at high C-rate $(1 / / h)$ differ strongly amongst these electrodes, although their porosity, thickness, and areal capacities are comparable (see Table II), clearly showing the significant effect of electrode tortuosity on the charging potential vs. SOC behavior, as would be expected based on theoretical models..$^{20,21}$

In addition to the difference in lithiation capacities at $11 / \mathrm{h}$, Figure $4 \mathrm{~b}$ also illustrates two other distinctive features in the lithiation potential vs. SOC profiles for increasing tortuosities, namely an increasing overpotential and a smearing out or complete loss of the typical potential steps in the graphite lithiation process at high C-rates. At the slow charging rate of $0.11 / \mathrm{h}$, the graphite lithiation plateaus (around SOC values of 10\%, 20\%, and 55\%) are clearly distinguishable and similarly well-defined for all graphite electrodes 
despite their vastly different tortuosities (compare Figure 4a). These plateaus are also still clearly visible at a C-rate of $11 / \mathrm{h}$ for the low tortuosity alginate based electrodes ( $\tau=3.1 \pm 0.1$, green, solid line). However, for electrodes with a higher tortuosity of $4.3 \pm 0.1$ (Kynar PVDF binder, dashed, orange line), the graphite potential plateaus are only partly visible and completely vanish for the electrode with the highest tortuosity ( $\tau=10.2 \pm 0.3, \mathrm{CMC} / \mathrm{SBR}$, pink line, compare Figure $4 b$ ). Both effects, the increasing overpotentials (depending on SOC, compare Figures $4 \mathrm{a}$ and $4 \mathrm{~b}$ ) as well as the degree of observable lithium intercalation plateaus are a result of ionic concentration gradients within the electrolyte phase across the electrode thickness, which builds up during the charging process. ${ }^{20,21}$ In this case lithium ions are intercalated into the graphite particles and are depleted in the electrolyte solution. At the same time, lithium ions generated at the counter electrode will be transported through the separator by migration and diffusion to the graphite anode/separator interface. If ionic transport through the electrode is fast compared to the selected charging time, i.e., if the electrode has a low tortuosity, the lithium ion concentration gradient across the graphite electrode remains low (i.e., the lithium ion concentration remains nearly constant) and the graphite electrode is charged homogeneously. In this case of nearly constant lithium ion concentration across the anode, the concentration overpotential also remains nearly constant across the anode thickness, so that the anode potential closely follows the graphite OCV curve or the charging curve a low C-rate (compare Figure 4a for low tortuosity alginate electrodes). On the other hand, for anodes with high tortuosity values, higher concentration gradients form across the anode electrode thickness, effectively leading to an inhomogeneous charging of the graphite electrode across its thickness. This, in turn, leads to the smearing out or the complete loss of the intercalation plateaus, as is best seen for the CMC/SBR electrode with the high tortuosity value (pink line in Figure $4 b$ ), an effect also reported from lithium ion battery simulations using different Bruggeman relations for the tortuosity of the electrode. ${ }^{21}$

The beneficial effect of lessened concentration gradients at $1 \mathrm{C}$ by reduced electrode tortuosities can also be seen from the capacities reached during a charging rate capability test. Mean values from three repeat cells for each electrode composition and their standard deviation (indicated by the error bars), are shown in Figure 5 for C-rates from $\sim 0.1 \mathrm{l} / \mathrm{h}$ to $20 \mathrm{1} / \mathrm{h}$ (charging capacities are again normalized to the $3^{\text {rd }}$ cycle charge capacity at $0.3 \mathrm{~mA} / A_{\text {El. }}$ (C-rate $\left.\sim 0.11 / \mathrm{h}\right)$ ). In line with the previously discussed potential transients at a C-rate of $\sim 11 / \mathrm{h}$, the graphite anodes with the lowest tortuosity show highest charge capacities at any given $\mathrm{C}$-rate.

The fit of a mathematical description to describe the sigmoidal experimental rate capability behavior was added as dashed lines in Figure 5 in order to provide a smooth guide-to-the-eye. Empirically, i.e., not based on any physics-based derivation we found the mathematical description

$$
y=\frac{y_{\max }}{1+\left(\frac{x}{x_{0}}\right)^{\text {slope }}}
$$

to be well-suited to describe the accessible capacity $Q_{\text {Acc. }}$ for all tortuosities and C-rates $R$. Applied to our measurements, $y_{\max }$ corresponds to the theoretical charging capacity in the low C-rate limit $(R \rightarrow 0), x$ becomes the $\mathrm{C}$-rate $R$, and the symmetry point $x_{0}$, i.e., the point where only $50 \%$ SOC are reversibly accessible is defined as critical C-rate, $R_{\text {crit. }}$. The slope $s$ remains as a free fitting parameter.

$$
Q_{\text {Acc. }}=\frac{Q_{\text {Theo. }}}{1+\left(\frac{R}{R_{\text {crit. }}}\right)^{\mathrm{s}}}
$$

The fit parameters $s$ and the obtained critical C-rates $R_{\text {crit. }}$ for the experimental data in Figure 5 are summarized in Table III.

For a tortuosity of $10.2 \pm 0.3,50 \%$ of the electrode's capacity can be utilized at a C-rate of $R_{\text {crit. }}=0.61 / \mathrm{h}$, while the same capacity is still accessible at $R_{\text {crit. }}=0.91 / \mathrm{h}$ for a tortuosity of $4.3 \pm 0.1$, and at $R_{\text {crit. }}=1.81 / \mathrm{h}$ for the lowest tortuosity of $3.1 \pm 0.1$. Thus, for

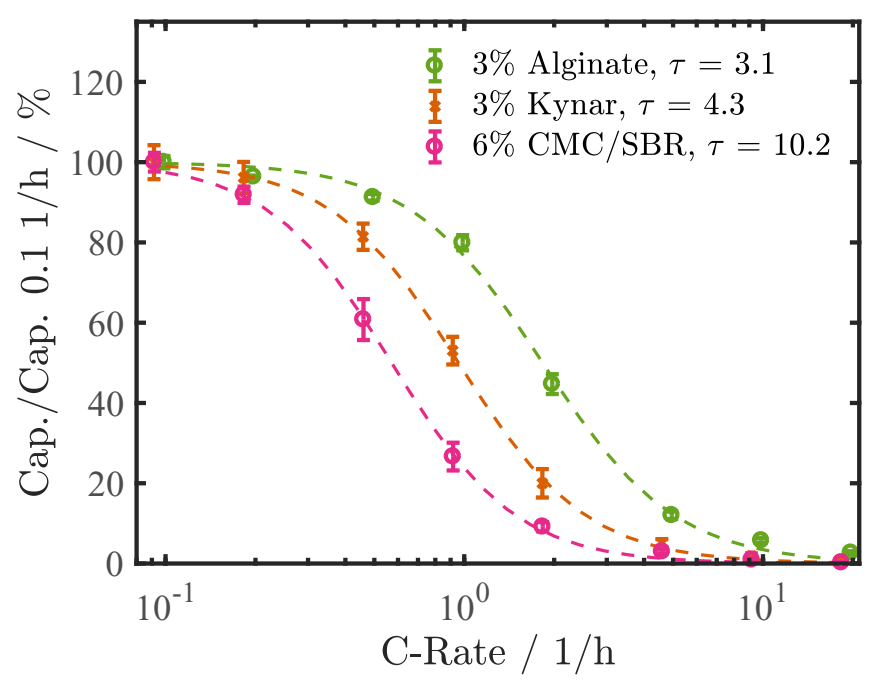

Figure 5. Mean charging (i.e., lithiation) capacities normalized to the capacity at $0.1 \mathrm{1} / \mathrm{h}$ of three types of electrodes with different tortuosities (see Table II; all areal capacities within $3.4 \pm 0.2 \mathrm{mAh} / \mathrm{cm}^{2}$ ) vs. galvanostatic charging rates ranging from $0.1 \mathrm{1} / \mathrm{h}$ to $20 \mathrm{1} / \mathrm{h}$, using a lower cutoff voltage of $5 \mathrm{mV}$ vs. a metallic lithium reference electrode. The discharge rate was kept constant at $0.1 \mathrm{1} / \mathrm{h}$ and the experiments were conducted at $25^{\circ} \mathrm{C}$ with $1 \mathrm{M} \mathrm{LiPF}_{6}$ in EC:EMC (3:7 w:w). Error bars indicate the standard error of the mean of three independent measurements with electrodes of identical composition. The dashed lines are the fit curves to Eq. 3, with the fitting parameters listed in Table III.

electrodes of similar mass, capacity, thickness and porosity (compare Table II), the C-rate at which the capacity drops below $50 \%$ can vary by a factor of three for electrode tortuosities ranging from $3.1 \pm 0.1$ to $10.2 \pm 0.3$. The experimentally found dependence of the critical C-rate on electrode tortuosity is in good agreement with the literature, where for a constant value of the accessible areal capacity the ratio of (dis)charge time for a full (dis)charge $t_{\mathrm{d}}$ over the tortuosity $\tau$ (i.e., $t_{d} / \tau$ ) would be predicted to remain constant (compare Eq. 14 in Ref. 20). Applied to our measurements this would mean that the product of the critical C-rate $R_{\text {crit. }}$ (corresponding to the inverse of the full (dis)charge time) and the tortuosity should remain constant.

$$
R_{\text {crit. }}^{\text {ideal }}=R_{\text {crit. }} \cdot \tau
$$

Here we refer to the factor of critical C-rate times tortuosity as the critical C-rate $\left(R_{\text {crit. }}^{\text {ideal }}\right)$ for an ideal electrode (in terms of tortuosity, this would mean an electrode with an ideal tortuosity of 1). Values for $R_{\text {crit. }}^{\text {ideal }}$, based on the critical C-rates and the tortuosity of the different electrodes are given Table III and are surprisingly similar. For the graphite electrodes with a tortuosity of 3.1 and 10.2 , closely agreeing $R_{\text {crit. }}$ values of $\sim 5.7$ and $\sim 5.8$ are obtained, while a slightly lower value of $\sim 3.9$ is obtained for the Kynar PVDF based anodes with a tortuosity of $4.3 \pm 0.1$. A somewhat smaller $R_{\text {crit. }}^{\text {ideal }}$ for the Kynar based PVDF electrodes might due to the generally larger variance of the measured charging capacity of the three repeat cells compared to the other binders (compare error bars of C-rate of 0.1 1/h in Figure 5).

Table III. Fitting parameters of Eq. 3 to the experimentally obtained capacities at C-rates from $\sim 0.11 / \mathrm{h}$ to $201 / \mathrm{h}$ and the calculated critical C-rates for the ideal electrode (using Eq. 4). The fitting curves are shown as dashed lines in Figure 5.

\begin{tabular}{cccccc}
$\begin{array}{c}\text { Composition } \\
\text { Gra/Bi/C65 }\end{array}$ & Binder & $\begin{array}{c}\text { Tortuosity } \\
-\end{array}$ & $\begin{array}{c}R_{\text {crit. }} \\
1 / \mathrm{h}\end{array}$ & $\begin{array}{c}R_{\text {crit. }}^{\text {ideal }} \\
1 / \mathrm{h}\end{array}$ & $\begin{array}{c}s \\
-\end{array}$ \\
\hline $97 / 3 / 0$ & Alginate & $3.1 \pm 0.1$ & 1.83 & 5.67 & 1.97 \\
$97 / 3 / 0$ & Kynar & $4.3 \pm 0.1$ & 0.90 & 3.88 & 2.25 \\
$94 / 6 / 0$ & CMC/SBR $1 / 1$ & $10.2 \pm 0.3$ & 0.57 & 5.83 & 2.09
\end{tabular}


It is noteworthy that for all electrode compositions the slope $s$ of the capacity loss in Eq. 3 is close to 2 (see Table III). By definition, the critical C-rate for the ideal electrode would correspond to a hypothetical electrode with a tortuosity of one (here somewhere between $\sim 4-61 / \mathrm{h})$. The reader is reminded that all cells are cycled at the same conditions, having the same thickness, porosity, and loading and that the same electrolyte is used. The constant value for $R_{\text {crit. }}^{\text {ideal }}$ thus shows that even for optimized graphite electrodes with, e.g., aligned particles, ${ }^{7}$ higher charging rates than $\sim 4-61 / \mathrm{h}$ may never be achieved for the given thickness, porosity, and electrolyte transport parameters. To confirm that in the theoretical scenario of a tortuosity of one the critical C-rate would range somewhere between $\sim 4-61 / \mathrm{h}$, numerical modeling studies would have to be conducted. However, here we focus on estimating the influence of the tortuosity on the accessible capacity at a given C-rate, which may be done using Eq. 3 and Eq. 4 with the fit parameters from Table III. We are aware that in general $R_{\text {crit. }}^{\text {ideal }}$ and $s$ will depend in some way on the electrolyte transport parameters and the slope of the OCV curve of the analyzed active material and emphasize that in this context Eq. 3 may only be used to understand the estimated performance change as a result of a tortuosity variation. Nevertheless, a rough estimate to predict the relative C-rate capability of anodes which differ only by their tortuosity value (i.e., which have identical thickness, porosity, and areal capacity) is afforded by Eq. 4 (e.g., doubling the tortuosity reduces the critical C-rate by a factor of 2). Since thickness, porosity, and areal capacity are typically electrode design requirements, simple and fast tortuosity measurements of electrodes in an electrode optimization study (e.g., variation of ink mixing and drying procedures) should thus serve as a rough predictor for the C-rate capability of electrodes.

\section{Conclusions}

Graphite electrodes with binder contents between 1.5 and $10 \mathrm{wt} \%$ of two water (CMC/SBR and alginate) and three NMP based (PVDF from Kynar, Kureha and Solef) binder systems have been prepared and analyzed in terms of their ionic tortuosities by analysis of the Nyquist plots of symmetric cell impedance measurements using a non-intercalating electrolyte with a simplified transmission line model equivalent circuit. ${ }^{3}$ Although the slurry mixing and electrode preparation procedure was not optimized, we find clear trends, i.e., higher tortuosities for higher amounts of binder and, more importantly, a wide range of tortuosity values for graphite electrodes of similar porosity and loading (from $2.7 \pm 0.1$ to $10.2 \pm 0.3$ ) for different types of polymeric binder. By addition of high surface area conductive carbon, the tortuosity of the electrodes with a binder layer thickness above $6 \mathrm{~nm}$ decreases.

Electrochemical charge performance tests of selected anodes with different tortuosities were done in three electrode cells with lithium counter and reference electrodes and showed a clear improvement, i.e., lower overpotentials and thus higher charge capacities, for low tortuosity electrodes. This is in line with the theoretically expected, reduced concentration gradients due to fast ionic transport through the porous anodes at low tortuosities. ${ }^{20,21}$ At last we describe an empirical expression which allows to estimate the accessible capacity for a given
C-rate and tortuosity and suggest that even for an ideal electrode, i.e., tortuosity of unity, the highest C-rate at which $50 \%$ of the nominal capacity can be accessed without lithium plating is $\sim 4-61 / \mathrm{h}$ for the here used anodes with a thickness of $\sim 100 \mu \mathrm{m}$, a porosity of $\sim 50 \%$, and an areal capacity of $\sim 3.4 \pm 0.2 \mathrm{mAh} / \mathrm{cm}^{2}$. The empirical expression introduced allows to roughly predict the dependence of the critical C-rate (at which $50 \%$ of the capacity is accessible) on the tortuosity of the electrode and thus serves as a valuable tool for electrode optimization studies.

\section{Acknowledgment}

We gratefully acknowledge the funding by the BMBF (Federal Ministry of Education and Research, Germany) for its financial support under the auspices of the ExZellTUM II project (grant number 03XP0081).

\section{ORCID}

Johannes Landesfeind (D) https://orcid.org/0000-0003-0333-2185

\section{References}

1. T. DuBeshter, P. K. Sinha, A. Sakars, G. W. Fly, and J. Jorne, J. Electrochem. Soc., 161, A599 (2014).

2. D. Cericola and M. E. Spahr, Electrochim. Acta, 191, 558 (2016)

3. J. Landesfeind, J. Hattendorff, A. Ehrl, W. A. Wall, and H. A. Gasteiger, J. Electrochem. Soc., 163, A1373 (2016)

4. I. V. Thorat, D. E. Stephenson, N. a. Zacharias, K. Zaghib, J. N. Harb, and D. R. Wheeler, J. Power Sources, 188, 592 (2009).

5. M. Ebner, D. W. Chung, R. E. García, and V. Wood, Adv. Energy Mater, 4, 1 (2014).

6. F. Tariq, V. Yufit, M. Kishimoto, P. R. Shearing, S. Menkin, D. Golodnitsky, J. Gelb, E. Peled, and N. P. Brandon, J. Power Sources, 248, 1014 (2014).

7. V. Wood and M. O. J. Ebner, EP Pat. App., EP20,130,001,974 (2014).

8. J. S. Kim, W. Pfleging, R. Kohler, H. J. Seifert, T. Y. Kim, D. Byun, H. G. Jung, W. Choi, and J. K. Lee, J. Power Sources, 279, 13 (2015),

9. S. Jaiser, M. Müller, M. Baunach, W. Bauer, P. Scharfer, and W. Schabel, J. Power Sources, 318, 210 (2016).

10. V. Barsykov and V. Khomenko, Sci. J. Riga Tech. Univ., 21, 67 (2010).

11. D. Guy, B. Lestriez, R. Bouchet, V. Gaudefroy, and D. Guyomard, J. Electrochem. Soc., 8, 17 (2005).

12. R. Wang, L. Feng, W. Yang, Y. Zhang, Y. Zhang, W. Bai, B. Liu, W. Zhang, Y. Chuan, Z. Zheng, and H. Guan, Nanoscale Res. Lett., 12, 575 (2017).

13. S. Komaba, N. Yabuuchi, T. Ozeki, Z. J. Han, K. Shimomura, H. Yui, Y. Katayama, and T. Miura, J. Phys. Chem. C, 116, 1380 (2012).

14. J. Landesfeind, M. Ebner, A. Eldiven, V. Wood, and H. A. Gasteiger, J. Electrochem. Soc., 165, A469 (2018)

15. T. Marks, S. Trussler, a. J. Smith, D. Xiong, and J. R. Dahn, J. Electrochem. Soc., 158, A51 (2011)

16. M. Müller, L. Pfaffmann, S. Jaiser, M. Baunach, V. Trouillet, F. Scheiba, P. Scharfer, W. Schabel, and W. Bauer, J. Power Sources, 340, 1 (2017).

17. H. Keiser, K. D. Beccu, and M. a. Gutjahr, Electrochim. Acta, 21, 539 (1976).

18. R. Morasch, B. Suthar, J. Landesfeind, and H. A. Gasteiger, in preparation (2018).

19. A. Ehrl, J. Landesfeind, W. A. Wall, and H. A. Gasteiger, J. Electrochem. Soc., 164, A826 (2017).

20. K. G. Gallagher, S. E. Trask, C. Bauer, T. Woehrle, S. F. Lux, M. Tschech, P. Lamp, B. J. Polzin, S. Ha, B. Long, Q. Wu, W. Lu, D. W. Dees, and A. N. Jansen, J. Electrochem. Soc., 163, 138 (2016).

21. B. Suthar, P. W. C. Northrop, D. Rife, and V. R. Subramanian, J. Electrochem. Soc., 162, 1708 (2015). 